\title{
¿Es la atrofia del hipocampo en la enfermedad de Parkinson un predictor de demencia?
}

\author{
Naroa Ibarretxe-Bilbao ${ }^{1}$ y Carme Junqué Plaja ${ }^{2}$ \\ 1 Personal Investigador Postdoctoral. Centro de Investigación Biomédica en Red de Enfermedades Neurodegenerativas \\ (CIBERNED). Sección Neurología. Hospital Clínic i Provincial de Barcelona. Departamento de Psiquiatría y Psicobiología \\ Clínica. Facultad de Medicina. Universitat de Barcelona. \\ 2Catedrática de Psicobiología. Departamento de Psiquiatría y Psicobiología Clínica. Facultad de Medicina. \\ Universitat de Barcelona. Jefe del grupo de Neuropsicología. Instituto de Investigaciones Biomédicas August Pi i Sunyer \\ (IDIBAPS). Barcelona.
}

\section{Resumen}

Fundamento y objetivo: la enfermedad de Parkinson (EP) es una enfermedad neurodegenerativa que conlleva frecuentemente el desarrollo de demencia. Entre los factores de riesgo para desarrollar una demencia en la EP, destacan las alucinaciones visuales (AV). En otras enfermedades neurodegenerativas, tales como la enfermedad de Alzheimer (EA), los estudios de resonancia magnética (RM) han demostrado que la presencia de atrofia del hipocampo en los sujetos con deterioro cognitivo leve es una medida predictora de evolución a demencia. El objetivo de este estudio es investigar el valor de la pérdida de sustancia gris del hipocampo como marcador de evolución a demencia en la EP. Pacientes y método: se evaluaron longitudinalmente $(30 \pm 5,7$ meses $)$ 11 pacientes con EP y AV $(\mathrm{EP}+\mathrm{AV})$ y 12 pacientes con $\mathrm{EP} \sin \mathrm{AV}(\mathrm{EP}-\mathrm{AV})$. Los pacientes fueron sometidos a una RM estructural y a una evaluación neuropsicológica al inicio del estudio y en el seguimiento. Los análisis individuales y grupales para evaluar la pérdida de sustancia gris del hipocampo se llevaron a cabo mediante regiones de interés (ROI) de la técnica voxel-based morphometry (VBM) implementada en el programa SPM5. Resultados: si bien inicialmente ninguno de los pacientes presentaba demencia, el $73 \%$ del grupo de EP + AV cumplía criterios de demencia en la exploración final. Ninguno de los pacientes sin AV desarrolló demencia. En el grupo de EP + AV, la atrofia del hipocampo evolucionó de un predominio anterior a anteroposterior, mientras que el grupo EP - AV no mostró pérdida de sustancia gris en esta estructura. En el análisis individual, el 64\% de los pacientes con AV presentaba atrofia del hipocampo y todos ellos desarrollaron demencia en el seguimiento. Conclusiones: la atrofia del hipocampo en la EP puede

\section{Abstract}

Background and objective: Parkinson's disease (PD) is a neurodegenerative disorder frequently associated to dementia. The presence of visual hallucinations $(V H)$ is a clinical predictor of dementia in PD. In other neurodegenerative diseases such as Alzheimer's disease (AD) magnetic resonance imaging (MRI) studies have shown that the atrophy of the hippocampus is a reliable predictor of evolution to dementia in subjects with amnesic mild cognitive impairment. The objective of the present study is to investigate the atrophy of the hippocampus as a predictor of dementia in PD. Patients and method: 11 PD patients with VH and 12 patients without $V H$ were longitudinally assessed after a follow-up period of $30 \pm 5.7$ months. Patients underwent a structural MRI and a neuropsychological assessment both at the entry of the study and at follow-up. Individual and group analyses to evaluate the hippocampal gray matter loss were carried out by means of region of interest analysis (ROI) from voxel-based morphometry (VBM) of the SPM5 program. Results: at base-line none of the patients met criteria for dementia. At follow-up $73 \%$ of the patients with VH met criteria for dementia whereas none of the patient without VM met criteria for it. In the PD with $V H$ group the hippocampal atrophy evolved from an anterior to antero-posterior predominance. The individual analyses showed that $64 \%$ of patients with VH presented hippocampal atrophy, and all of those who presented atrophy developed dementia. Conclusion: the hippocampal atrophy in PD is a marker of evolution to dementia and can explain the memory decline observed in these patients.

(Alzheimer. Real Invest Demenc. 2011;49:5-11) 
constituir un marcador objetivo de evolución a demencia y puede explicar el declive de memoria descrito en estos pacientes.

(Alzheimer. Real Invest Demenc. 2011;49:5-11)

Palabras clave: enfermedad de Parkinson, demencia, resonancia magnética, hipocampo, memoria.
Keywords: Parkinson's disease, dementia, magnetic resonance imaging, hippocampus, memory.

\section{Introducción}

La enfermedad de Parkinson (EP) ha sido tradicionalmente considerada un trastorno exclusivamente motor caracterizado por temblor, rigidez, bradicinesia e inestabilidad postural. Sin embargo, los síntomas no motores, tales como el deterioro cognitivo y el desarrollo de demencia, frecuentemente acompañan el curso de la enfermedad ${ }^{1}$.

El deterioro cognitivo en la EP comienza desde los estadios iniciales, y la memoria, las funciones ejecutivas y las funciones visuoperceptivas son las funciones cognitivas más alteradas ${ }^{2-5}$. A medida que avanza la enfermedad, un gran número de pacientes desarrollará demencia, y el $83 \%$ de los supervivientes a 20 años serán los que cumplan criterios para el diagnóstico de demencia ${ }^{6}$.

Entre los factores de riesgo o predictores de demencia, se encuentran las alucinaciones visuales $(\mathrm{AV})^{7,8}$. La prevalencia de las AV se sitúa alrededor del $50 \%{ }^{9}$, generalmente aparecen en la segunda mitad del curso de la enfermedad y son de naturaleza persistente y progresiva. Tradicionalmente, las AV habían sido descritas como una complicación secundaria a la medicación antiparkinsoniana dopaminérgica. No obstante, cada vez son más los datos que señalan que otros factores, como la extensión del proceso neuropatológico a áreas temporales mediales del cerebro, podrían ser los desencadenantes ${ }^{9}$.

Recibido para su publicación: 2 de julio de 2010.

Aceptado para su publicación: 21 de julio de 2010.

Correspondencia: Carme Junqué Plaja.

E-mail: cjunque@ub.edu
Los pacientes con AV, en comparación con los pacientes sin ellas, manifiestan un mayor déficit en la memoria verbal ${ }^{10,11}$. Si bien los estudios iniciales clásicos proponían que la causa del déficit de memoria en la EP era la disfunción del circuito frontoestriatal a causa de la depleción dopaminérgica ${ }^{12}$, los estudios neuropatológicos ${ }^{13-15}$ y los estudios recientes de resonancia magnética (RM) estructural ponen de manifiesto la implicación de la atrofia del hipocampo en la disfunción de la memoria en la EP16-20.

Nuestro grupo recientemente publicó un estudio en el que se observó que los pacientes con EP y demencia presentaban una atrofia del hipocampo que abarcaba regiones anteriores y posteriores de esta estructura preservando las partes mediales. Los pacientes no dementes con $\mathrm{AV}$ y, por tanto, con un mayor riesgo de desarrollar demencia, presentaban atrofia hipocampal en la parte anterior de dicha estructura. Los pacientes sin $\mathrm{AV}$ y, por tanto, con menor factor de riesgo de evolución a demencia, no presentaron atrofia hipocampal en este estudio inicial ${ }^{20}$.

El presente trabajo es un estudio longitudinal de la muestra anterior cuyo objetivo es estudiar la evolución de los déficits de memoria y la progresión a demencia en pacientes con EP con y sin AV. Pretendemos, además, investigar el valor de la atrofia progresiva del hipocampo como marcador de evolución a demencia en la EP. Establecimos la hipótesis de que los pacientes con EP y AV presentarán desarrollo de demencia y un marcado deterioro en las funciones de memoria verbal, y que la atrofia cerebral en los pacientes con AV que han evolucionado a demencia abarcará el hipocampo de una manera y con un patrón similar a lo encontrado en los pacientes con demencia. 


\section{Pacientes y método}

La muestra de este trabajo forma parte de un proyecto dedicado al estudio de pacientes con factores de riesgo de demencia en la EP (en concreto, pacientes con AV) llevado a cabo por el grupo de Neuropsicología del Departamento de Psiquiatría y Psicobiología Clínica de la Universidad de Barcelona, en colaboración con la Unidad de Parkinson y Trastornos del Movimiento del Hospital Clínic de Barcelona.

El estudio inicial ${ }^{20}$ estaba compuesto de 46 pacientes: 9 pacientes con EP y demencia, 16 pacientes con EP y AV, 19 pacientes con EP sin AV y 56 controles. Los dos grupos de pacientes estaban apareados por edad, sexo y años de educación. Después de un período de seguimiento de 30 meses (desviación estándar: 5,74), 11 pacientes con AV y 12 pacientes sin AV accedieron a participar en el estudio. En el estudio longitudinal no participó ninguno de los pacientes con EP y demencia debido a su estado deteriorado. Las imágenes de RM de los 56 controles fueron utilizadas tanto en el estudio inicial como en el longitudinal para construir una plantilla normativa con la cual comparar las imágenes de los pacientes. El estudio fue aprobado por el Comité Ético de la Universidad de Barcelona y todos los participantes firmaron el consentimiento informado.

El estudio inicial y de seguimiento consistían en:

1. Evaluación de la presencia de AV, las cuales fueron evaluadas mediante una entrevista estructurada desarrollada en nuestro hospital que cubre aspectos referentes a la modalidad (visual, auditiva, táctil y olfativa), contenido (animales, personas, objetos) y temporalidad (momento del día, frecuencia y duración). La gravedad de las AV fue estimada mediante el inventario neuropsiquiátrico de Cummings (subescala de alucinaciones). Las AV de nuestra muestra eran visuales y consistían en imágenes bien formadas de animales y/o personas, que ocurrían mientras los pacientes estaban alerta y con los ojos abiertos. Los pacientes eran conscientes de que se trataba de una alucinación en la mayoría de los casos y no presentaban paranoia asociada al fenómeno.
2. Diagnóstico de demencia, que se efectuó en función de los criterios propuestos por la Sociedad Internacional de Trastornos del Movimiento ${ }^{21}$. El algoritmo para el diagnóstico de demencia según estos criterios requiere: $a$ ) diagnóstico de EP; $b$ ) desarrollo de síntomas motores de la EP previo a la aparición de demencia; c) Mini-Mental State Examination (MMSE) por debajo de 26; d) presencia de déficits cognitivos lo suficientemente incapacitantes para alterar las actividades de la vida diaria, y e) alteración en uno o más de los dominios cognitivos incluyendo atención, función ejecutiva, habilidades visuoconstructivas o memoria.

3. Evaluación específica de la memoria verbal mediante el test de memoria auditivoverbal de Rey. Las variables de memoria evaluadas incluyeron: aprendizaje (suma de recuerdo tras cinco ensayos consecutivos), recuerdo demorado al de 20 minutos y el reconocimiento.

4. Adquisición de imágenes de RM estructural potenciadas en T1 con una máquina de RM de 1,5 tesla (General Electric) en el Centre de Diagnòstic per la Imatge (CDIC), del Hospital Clínic de Barcelona. El protocolo de adquisición incluía una secuencia axial de imágenes estructurales 3D con los siguientes parámetros: TR (tiempo de repetición $)=17$; TE $($ tiempo de eco $)=5$; TI (tiempo de inversión $)=300$; 1,5 mm de grosor; campo de visión $=24 \times 24$; matriz = $256 \times 256$; 1 NEX (número de excitaciones). Las imágenes de RM se analizaron mediante la técnica de voxel-based morphometry $(\mathrm{VBM})^{22}$ para evaluar las diferencias grupales y la pérdida de sustancia gris en el hipocampo. El procesamiento de las imágenes se realizó mediante el software SPM5 (Statistical Parametric Mapping; Wellcome Department of Cognitive Neurology, University College London, UK, http://www.fil.ion.ucl.ac.uk/spm) con Matlab 7.0 (MathWorks, Natick, MA). Primero, las imágenes se reorientaron de acuerdo con la comisura anterior y posterior, y luego fueron segmentadas en sustancia gris, sustancia blanca y líquido cefalorraquídeo. Las imágenes de sustancia gris fueron normalizadas a la plantilla del SPM y luego fueron suavizadas con un kernel gaussiano de $6 \mathrm{~mm}$. Ya que 
nuestro interés estaba centrado en analizar los cambios en el hipocampo, se utilizó una máscara de dicha estructura obtenida a partir del atlas WFU-pickatalas diseñado para la presente versión del SPM. Todos los resultados fueron corregidos a nivel de clúster con una $\mathrm{p}<0,05$ corregida por múltiples comparaciones usando FDR (False Discovery Rate).

\section{Resultados}

\section{Deterioro de la memoria verbal y evolución a demencia}

El 73\% de los pacientes del grupo EP con AV, que inicialmente eran no dementes, cumplieron criterios para el diagnóstico de demencia en el seguimiento. Del grupo de los EP sin AV, ninguno presentó evolución a demencia.

En la tabla 1 se muestran las puntuaciones en las variables clínicas y de memoria al inicio del estudio y en el seguimiento. Tanto los pacientes con AV como los pacientes sin AV mostraron mayores puntuaciones en la sección motora de la Unified Parkinson Disease Rating Scale (UPDRS), lo cual indicaba un empeoramiento de los síntomas motores con el tiempo en ambos grupos. Sin embargo, sólo los pacientes con AV mostraron un deterioro progresivo en el MMSE y en variables de memoria verbal, tales como el aprendizaje y el recuerdo demorado.

\section{Comparaciones grupales con VBM}

En el estudio de seguimiento, los pacientes con EP y AV en comparación con los controles mostraron reducción de la sustancia gris que abarca las partes anterior y posterior de dicha estructura (tabla 2). En la figura 1 se presentan los cortes coronales en los que aparece el hipocampo, siguiendo los cortes que, de acuerdo con otros estudios, abarcan el hipocampo ${ }^{20,23}$; los cortes desde -40 a -34 corresponden a la parte más posterior del hipocampo (véase la cola); los cortes desde -32 a -18 corresponden al cuerpo del hipocampo y los cortes desde -16 a -4 corresponden a la parte anterior o cabeza del hipocampo. Los pacientes con EP y AV mostraban atrofia en la cabeza y en la cola. En con-

\begin{tabular}{|l|c|c|c|c|c|}
\hline & & Estudio inicial & Estudio seguimiento & t test & p* \\
\hline Test depresión & EP con AV: & $7,64 \pm 4,27$ & $8,91 \pm 6,41$ & $-0,645$ & 0,533 \\
de Hamilton & EP sin AV: & $3,33 \pm 3,05$ & $4,75 \pm 5,43$ & $-0,884$ & 0,396 \\
\hline Hoehn y Yahr & EP con AV: & $3,09 \pm 1,15$ & $3,68 \pm 1,27$ & $-2,358$ & $0,040^{*}$ \\
& EP sin AV: & $2,29 \pm 0,54$ & $2,75 \pm 0,94$ & $-1,570$ & 0,144 \\
\hline UPDRS III & EP con AV: & $26,45 \pm 12,40$ & $42,55 \pm 20,81$ & $-2,272$ & $0,020^{*}$ \\
& EP sin AV: & $22,25 \pm 13,70$ & $31,08 \pm 12,97$ & $-2,90$ & $0,014^{*}$ \\
\hline MMSE & EP con AV: & $26,64 \pm 1,74$ & $19,73 \pm 7,53$ & 3,637 & $0,005^{*}$ \\
& EP sin AV: & $29,33 \pm 1,61$ & $29,08 \pm 0,67$ & 5,221 & 0,612 \\
\hline TAVR & EP con AV: & $27,00 \pm 6,67$ & $20,18 \pm 8,97$ & 3,034 & $0,013^{*}$ \\
Aprendizaje & EP sin AV: & $41,25 \pm 4,92$ & $42,83 \pm 7,79$ & $-0,781$ & 0,451 \\
\hline TAVR & EP con AV: & $5,20 \pm 1,68$ & $3,00 \pm 2,10$ & 3,161 & $0,012^{*}$ \\
Recuerdo demorado & EP sin AV: & $9,00 \pm 1,60$ & $10,17 \pm 2,40$ & $-1,984$ & 0,073 \\
\hline TAVR & EP con AV: & $12,70 \pm 1,25$ & $12,40 \pm 2,11$ & 0,380 & 0.713 \\
Reconocimiento & EP sin AV: & $13,75 \pm 1,48$ & $13,92 \pm 1,67$ & $-0,364$ & 0.723 \\
\hline
\end{tabular}

Tabla 1. Características clínicas y rendimiento en memoria de los pacientes con enfermedad de Parkinson en el estudio inicial y el seguimiento.

*Diferencias significativas $(p<0,05)$ entre estudio inicial y seguimiento.

AV: alucinaciones visuales; EP: enfermedad de Parkinson; MMSE: Mini-Mental State Examination; TAVR: test de aprendizaje auditivoverbal de Rey; UPDRS III: Unified Parkinson Disease Rating Scale (sección motora). 


\begin{tabular}{|l|ccc|c|c|c|}
\hline \multirow{2}{*}{ Estructura } & \multicolumn{2}{|c|}{ Coordenadas MNI* } & Tamaño del clúster & puntuación $\mathbf{t}$ & p corregida \\
\hline Cabeza hipocampo izquierda & -28 & -8 & -14 & 210 & 6,00 & 0,016 \\
\hline Cabeza hipocampo derecha & -18 & -34 & 0 & 172 & 3,78 & 0,030 \\
\hline Cola hipocampo izquierda & 22 & -6 & -18 & 212 & 4,60 & 0,030 \\
\hline Cola hipocampo derecha & 20 & -36 & 4 & 144 & 3,74 & 0,050 \\
\hline
\end{tabular}

Tabla 2. Diferencias entre los pacientes con enfermedad de Parkinson con alucinaciones visuales y los controles en el seguimiento.

MNI: Montreal Neurological Institute.

* Las coordenadas MNI se refieren al vóxel de máxima significación dentro del clúster.

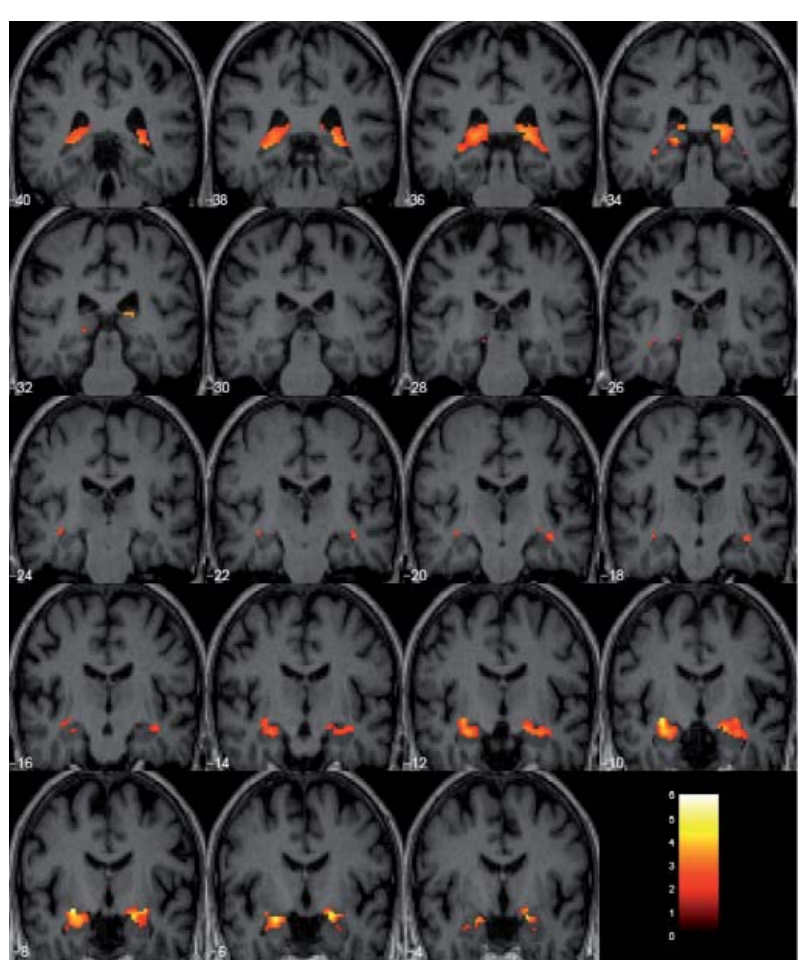

Figura 1. Las imágenes coloreadas representan la superficie donde se observa una pérdida de sustancia gris en el hipocampo en los pacientes con enfermedad de Parkinson y alucinaciones visuales, durante un seguimiento de aproximadamente 30 meses. La atrofia del hipocampo afecta a la parte anterior (cabeza) y posterior (cola). La barra de colores indica el grado de significación de los valores de t; el color amarillo es más estadísticamente significativo que el rojo.

traste, el cuerpo del hipocampo está relativamente preservado. Los pacientes con EP sin AV no mostraron atrofia del hipocampo en el estudio de seguimiento en comparación con los controles.

\section{Análisis individuales}

Del grupo de EP con AV, 7 pacientes (64\%) mostraron pérdida de sustancia gris en el hipocampo en el estudio inicial y la mantuvieron en el seguimiento, durante el cual todos ellos desarrollaron demencia.

Del grupo EP sin AV, sólo 3 pacientes (25\%) mostraron atrofia del hipocampo. Y si bien estos pacientes no cumplían criterios diagnósticos de demencia, obtuvieron peores puntuaciones en aprendizaje en comparación con aquellos que no mostraban atrofia del hipocampo $(\mathrm{t}=3,71 ; \mathrm{p}<0,004)$.

\section{Discusión}

Los pacientes con EP y con factores de riesgo de evolución a demencia (en este caso, presencia de AV) presentan un alto porcentaje de casos $(73 \%)$ que evolucionan a demencia tras un período de seguimiento de 30 meses y un mayor deterioro de la memoria verbal en comparación con los pacientes sin AV. La atrofia del hipocampo en el grupo de EP y AV, que en el estudio inicial sólo afectaba a la cabeza del hipocampo ${ }^{20}$, en el estudio de seguimiento afectaba a la parte anterior y posterior del hipocampo, lo cual se correspondía con el patrón de atrofia previamente descrito en sujetos con EP y demencia. En contraste con la evolución de los pacientes con AV, los pacientes sin AV no mostraron evolución a demencia y no se observó pérdida progresiva de sustancia gris en el hipocampo. 
Por tanto, aunque la atrofia de estructuras temporales mediales sea una característica principal de la enfermedad de Alzheimer ${ }^{24,25}$, también parece subyacer a la presencia de deterioro cognitivo y evolución a demencia en la EP. De hecho, los pacientes con AV de nuestro estudio presentaban atrofia de la cabeza del hipocampo previamente al desarrollo de demencia. La cabeza del hipocampo se corresponde básicamente con la región CA1, y esta región es especialmente vulnerable a cambios de tipo Alzheimer, incluso en las fases iniciales de la enfermedad 26,27 . Además, se ha descrito que la reducción del volumen del hipocampo y, especialmente, la implicación del sector CAl están asociadas a un mayor riesgo de conversión de deterioro cognitivo leve a demencia de tipo Alzheimer ${ }^{28}$. Tras el seguimiento, los pacientes con AV evolucionaron a demencia y además mostraron un patrón de atrofia más extendido que afectaba a partes anteriores y posteriores del hipocampo. Por otro lado, los análisis individuales también mostraron que el hecho de presentar atrofia del hipocampo en el estudio inicial está asociado con una mayor probabilidad de desarrollar demencia y/o deterioro de la memoria verbal en el seguimiento.

No podemos comprobar mediante neuroimagen estructural si el sustrato patológico de la atrofia hipocampal descrita en este estudio se debe a cambios tipo Alzheimer o de cuerpos de Lewy; pero es importante tener en cuenta que la demencia en la EP también es dependiente de cambios patológicos tipo Alzheimer, habiéndose descrito la presencia de ovillos neurofibrilares y placas de beta-amiloide en pacientes con EP y demencia ${ }^{29}$. Seguramente ambas enfermedades desempeñan un papel importante en el desarrollo de la atrofia del hipocampo y, por tanto, en la evolución a demencia y afectación de la memoria verbal.

En conclusión, los resultados de este estudio demuestran que la presencia de atrofia del hipocampo podría considerare como un índice predictor de evolución a demencia en la EP en pacientes con $\mathrm{AV}$.

\section{Bibliografía}

1. Aarsland D, Zaccai J, Brayne C. A systematic review of prevalence studies of dementia in Parkinson's disease. Mov Disord. 2005;20:1255-63.

2. Foltynie T, Brayne CE, Robbins TW, Barker RA. The cognitive ability of an incident cohort of Parkinson's patients in the UK. The CamPaIGN study. Brain. 2004;127:55060 .

3. Muslimovic D, Post B, Speelman JD, Schmand B. Cognitive profile of patients with newly diagnosed Parkinson disease. Neurology. 2005;65:1239-45.

4. Aarsland D, Bronnick K, Larsen JP, Tysnes OB, Alves G, Norwegian ParkWest Study Group. Cognitive impairment in incident, untreated Parkinson disease: the Norwegian ParkWest study. Neurology. 2009;72:1121-6.

5. Elgh E, Domellöf M, Linder J, Edstrom M, Stenlund H, Forsgren L. Cognitive function in early Parkinson's disease: a population-based study. Eur J Neurol. 2009; 16:127884.

6. Hely MA, Reid WG, Adena MA, Halliday GM, Morris JG. The Sydney multicenter study of Parkinson's disease: the inevitability of dementia at 20 years. Mov Disord. 2008;23:837-44.

7. Aarsland D, Andersen K, Larsen JP, Lolk A, KraghSorensen P. Prevalence and characteristics of dementia in Parkinson disease: an 8-year prospective study. Arch Neurol. 2003;60:387-92.

8. Galvin JE, Pollack J, Morris JC. Clinical phenotype of Parkinson disease dementia. Neurology. 2006;67:160511.

9. Williams DR, Lees AJ. Visual hallucinations in the diagnosis of idiopathic Parkinson's disease: a retrospective autopsy study. Lancet Neurol. 2005;4:605-10.

10. Ramírez-Ruiz B, Junque C, Martí MJ, Valldeoriola F, Tolosa E. Neuropsychological deficits in Parkinson's disease patients with visual hallucinations. Mov Disord. 2006;21:1483-7.

11. Santangelo G, Trojano L, Vitale C, Ianniciello M, Amboni M, Grossi D, et al. A neuropsychological longitudinal study in Parkinson's patients with and without hallucinations. Mov Disord. 2007;22:2418-25.

12. Cooper JA, Sagar HJ, Jordan N, Harvey NS, Sullivan EV. Cognitive impairment in early, untreated Parkinson's disease and its relationship to motor disability. Brain. 1991; 114(Pt 5):2095-122.

13. Churchyard A, Lees AJ. The relationship between dementia and direct involvement of the hippocampus and amygdala in Parkinson's disease. Neurology. 1997;49:1570-6.

14. Apaydin H, Ahlskog JE, Parisi JE, Boeve BF, Dickson DW. Parkinson disease neuropathology: later-developing dementia and loss of the levodopa response. Arch Neurol. 2002;59:102-12. 
15. Braak H, Del Tredici K, Rub U, De Vos RA, Jansen Steur EN, Braak E. Staging of brain pathology related to sporadic Parkinson's disease. Neurobiol Aging. 2003;24:197-211.

16. Camicioli R, Moore MM, Kinney A, Corbridge E, Glassberg K, Kaye JA. Parkinson's disease is associated with hippocampal atrophy. Mov Disord. 2003;18:784-90.

17. Bruck A, Kurki T, Kaasinen V, Vahlberg T, Rinne JO. Hippocampal and prefrontal atrophy in patients with early non-demented Parkinson's disease is related to cognitive impairment. J Neurol Neurosurg Psychiatry. 2004;75: 1467-9.

18. Junque C, Ramírez-Ruiz B, Tolosa E, Summerfield C, Martí MJ, Pastor P, et al. Amygdalar and hippocampal MRI volumetric reductions in Parkinson's disease with dementia. Mov Disord. 2005;20:540-4.

19. Bouchard TP, Malykhin N, Martín WR, Hanstock CC, Emery DJ, Fisher NJ, et al. Age and dementia-associated atrophy predominates in the hippocampal head and amygdala in Parkinson's disease. Neurobiol Aging. 2008;29: 1027-39.

20. Ibarretxe-Bilbao N, Ramírez-Ruiz B, Tolosa E, Martí MJ, Valldeoriola F, Bargallo N, et al. Hippocampal head atrophy predominance in Parkinson's disease with hallucinations and with dementia. J Neurol. 2008;255:1324-31.

21. Dubois B, Burn D, Goetz C, Aarsland D, Brown RG, Broe GA, et al. Diagnostic procedures for Parkinson's disease dementia: recommendations from the movement disorder society task force. Mov Disord. 2007;22:2314-24.

22. Ashburner J, Friston KJ. Voxel-based morphometry: the methods. Neuroimage. 2000;1 1:805-21.
23. Hackert VH, Den Heijer T, Oudkerk M, Koudstaal PJ, Hofman A, Breteler MM. Hippocampal head size associated with verbal memory performance in nondemented elderly. Neuroimage. 2002;17:1365-72.

24. Scheltens P, Leys D, Barkhof F, Huglo D, Weinstein HC, Vermersch P, et al. Atrophy of medial temporal lobes on MRI in «probable» Alzheimer's disease and normal ageing: diagnostic value and neuropsychological correlates. J Neurol Neurosurg Psychiatry. 1992;55:967-72.

25. Scheltens P, Fox N, Barkhof F, De Carli C. Structural magnetic resonance imaging in the practical assessment of dementia: beyond exclusion. Lancet Neurol. 2002;1: $13-21$.

26. Frisoni GB, Testa C, Zorzan A, Sabattoli F, Beltramello A, Soininen $\mathrm{H}$, et al. Detection of grey matter loss in mild Alzheimer's disease with voxel based morphometry. J Neurol Neurosurg Psychiatry. 2002;73:657-64.

27. Wang L, Swank JS, Glick IE, Gado MH, Miller MI, Morris JC, et al. Changes in hippocampal volume and shape across time distinguish dementia of the Alzheimer type from healthy aging. Neuroimage. 2003;20:667-82.

28. Apostolova LG, Dutton RA, Dinov ID, Hayashi KM, Toga AW, Cummings JL, et al. Conversion of mild cognitive impairment to Alzheimer disease predicted by hippocampal atrophy maps. Arch Neurol. 2006;63:693-9.

29. Jellinger KA, Seppi K, Wenning GK, Poewe W. Impact of coexistent Alzheimer pathology on the natural history of Parkinson's disease. J Neural Transm. 2002;109:329-39. 